\title{
Evidence for anomalous optical transition radiation linear polarization effects in beam-profile monitors
}

\author{
A. H. Lumpkin, ${ }^{*}$ A. S. Johnson, J. Ruan, and R. M. Thurman-Keup \\ Fermi National Accelerator Laboratory, Batavia, Illinois 60510, USA \\ C.-Y. Yao \\ Argonne National Laboratory, Argonne, Illinois 60439, USA \\ P. Evtushenko \\ Thomas Jefferson National Laboratory, Newport News, Virginia 23606, USA
}

(Received 26 April 2013; published 8 October 2013)

\begin{abstract}
Investigations of the effects of optical transition radiation (OTR) polarization components on beam profiles are presented. The transverse profiles are examined using the OTR perpendicular and parallel polarization components with respect to the dimension of interest. We observed $\sim 15 \%$ projected profile size reductions with the perpendicularly polarized components on a $65-\mu \mathrm{m}$ beam image size case at $14 \mathrm{MeV}$, a $150-\mu \mathrm{m}$ beam image size at $4.5 \mathrm{GeV}$, and a $1100-\mu \mathrm{m}$ beam image size at $7 \mathrm{GeV}$. These effects are all several times larger than expected (and anomalous in this sense) when compared to the standard OTR point-spread function calculations. We propose the time-averaged induced-current distribution which generates the OTR represents the actual beam size more faithfully with the perpendicular polarization component and recommend its routine use and subsequent deconvolution.
\end{abstract}

\section{INTRODUCTION}

The characterization of the transverse beam size of relativistic electron beams using optical transition radiation (OTR) imaging [1-4] has been implemented at many accelerators in the past two decades. OTR imaging had been considered a reliable method for obtaining beam profiles [5-9] prior to the identification of the microbunching instability and its concomitant coherent intensity fluctuations that are evident in many compressed bright beams [10-14]. Besides that issue, theoretical modeling has identified only minor polarization effects in the point-spread function (PSF) [6] or single-particle function [7]. However, we examine empirical evidence [15-19] that the utilization of the polarization component orthogonal to the dimension of interest results in a noticeably smaller observed projected image profile than theoretically predicted [5-9]. In those calculations the OTR point-spread-function width was in the few-micron range for 0.10 rad collection angles so only experiments that involve beam sizes with sigma $<20$ microns might be concerned. In practice most beamprofiling experiments have not considered the OTR polarization effects, investigators did not install linear polarizers in their optical transport lines, and their effective collection

\footnotetext{
*lumpkin@fnal.gov

Published by the American Physical Society under the terms of the Creative Commons Attribution 3.0 License. Further distribution of this work must maintain attribution to the author(s) and the published article's title, journal citation, and DOI.
}

angle was reduced by a final lens stop. A previously unpublished OTR result at $7 \mathrm{GeV}$ on a transport line exhibited apparent polarization effects at the $1000-\mu \mathrm{m}$ regime although only the optical diffraction radiation (ODR) polarization results were explicitly reported [17]. These OTR data are now reported, as well as a similar effect within the OTR/ODR tests at Jefferson National Lab (JLAB) at $4.5 \mathrm{GeV}$ in the $125-$ to $300-\mu \mathrm{m}$ regime [18].

We have recently continued investigations of this phenomenon at low gamma at Fermilab with a more controlled experiment where the linear polarizers are selectable in a filter wheel which also includes a clear glass position to compensate for the optical path. The aperture for light collection at the polarizer position is thus kept fixed compared to the previous tests at Fermilab and JLAB $[15,18]$. At the relatively low gamma of $\sim 30$, the horizontal polarization component of OTR is more asymmetric than the vertical one in our optical solid angle. We still observed $11 \%-18 \%$ projected profile size reductions on a $65-\mu \mathrm{m}$ beam-size case with the perpendicularly polarized components. This similar anomalous effect of $\sim 15 \%$ (which was observed over 2 orders of magnitude in gamma and a factor of 20 in beam size in these collected experiments) is compared to results from a standard OTR point-spreadfunction (PSF) model [5-9]. The potential for overestimating beam sizes with OTR imaging is addressed in this paper.

\section{EXPERIMENTAL ASPECTS}

The most recent tests were performed at the Fermilab A0 photoinjector (A0PI) facility which includes an L-band 


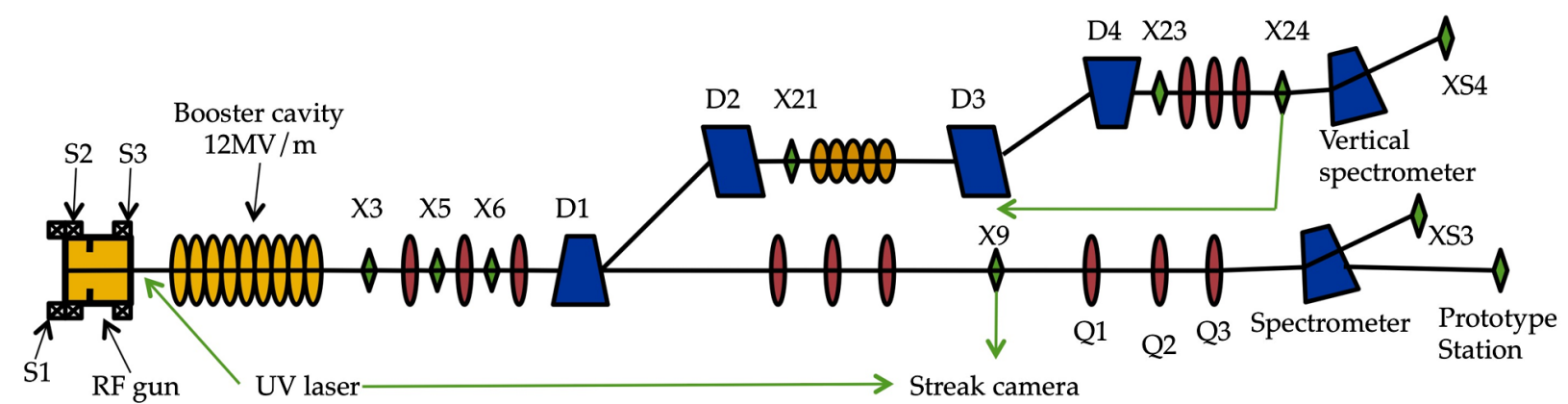

FIG. 1. A schematic of the A0 photoinjector test area showing the PC $\mathrm{rf}$ gun, nine-cell booster cavity, transverse emittance stations, and the OTR stations. The X5 and prototype station locations are indicated.

photocathode (PC) rf gun and a nine-cell superconducting rf accelerating structure which combine to generate up to $16-\mathrm{MeV}$ electron beams. The drive laser operates at $81.25 \mathrm{MHz}$ although the micropulse structure is divided down to $1 \mathrm{MHz}$ rate. Because of the low electron-beam energies and low OTR signals, we typically summed over micropulses depending on the charge per micropulse. Micropulse charges from 25 to $500 \mathrm{pC}$ were used for beam sigma sizes of 45 to 250 microns. Initial experiments were performed at station X5 comparing OTR with a powder scintillator $[19,20]$. Subsequently, the prototype station was installed in the user beam line section beyond the horizontal spectrometer in the straight ahead line as indicated in Fig. 1. The nominal beam parameters are given in Table I. We integrated signal over 10-50 micropulses to obtain adequate OTR signal.

The prototype station (see Fig. 2) consists of a vacuum cross with a three-position pneumatic actuator allowing selection of a beam-impedance matching screen, a $100-\mu \mathrm{m}$ thick YAG : Ce single crystal with its surface normal to the beam direction followed by a $45^{\circ}$ turning mirror, or a $1-\mu \mathrm{m}$ thick $\mathrm{Al}$ foil for OTR followed by a $45^{\circ}$ turning mirror. For the OTR polarization tests we removed the thin first foil and used the turning mirror as the OTR screen. We refocused the optics by translating the optical assembly back from the viewing window by $12.5 \mathrm{~mm}$ so the center of this OTR screen was in focus. For these tests both turning mirrors were an aluminized $\mathrm{Si}$ substrate (200 $\mu \mathrm{m}$ thick). As part of the optics design, a backilluminated virtual target option with matched field lens could be selected by inserting a beam splitter into the relay

TABLE I. Summary of nominal electron-beam parameters for operations at A0PI with $250 \mathrm{pC}$ per micropulse and a drive laser bunch length of $2.7 \mathrm{ps}$ (sigma).

\begin{tabular}{lcc}
\hline \hline Parameter & Units & Value \\
\hline Energy & $\mathrm{MeV}$ & 15 \\
Energy spread & $\mathrm{keV}$ & $10-15$ \\
Transverse emittance & $\mathrm{mm} \mathrm{mrad}$ & $2.6 \pm 0.3$ \\
Bunch length $(\sigma)$ & $\mathrm{ps}$ & $3.1 \pm 0.3$ \\
\hline \hline
\end{tabular}

optics path. This scene was then relayed to the final Computar zoom lens mounted on the 1.3 Megapixel Prosilica CCD camera and used for resolution and optics calibration aspects. The optical resolution tests were reported previously [16]. A filter wheel was used to select neutral density filters or one of the two linear polarizers which were oriented with the axes in the horizontal and vertical directions. This prototype station was constructed by RadiaBeam Technologies under a contract with Fermilab.

The optical system had $7-\mu \mathrm{m}$ rms spatial resolution in the central 2-mm region when covering a vertical field of view of $5 \mathrm{~mm}$. The calibration factor was $5.3 \mu \mathrm{m}$ per pixel. Because of the size of the polarization effects, such resolution was advantageous. Recently, we verified the collection angle of the prototype optics by using a laser source mounted on a small rotation stage at the screen object distance. The first field lens of $50 \mathrm{~mm}$ diameter was $135 \mathrm{~mm}$ from the source point resulting in a half angle of $160 \mathrm{mrad}$, However, the $C$-mount zoom lens at the CCD was shown to reduce the effective angle to $80 \mathrm{mrad}$ when at fully open iris. We closed the iris less than one stop which reduced the OTR intensity, and estimate the final effective angle was 50 to $60 \mathrm{mrad}$.

The tests at JLAB were performed in one extraction line to a nuclear physics test area in Hall $\mathrm{A}$ of the Continuous Electron Beam Accelerator Facility (CEBAF) and were motivated by an interest in optical diffraction radiation (ODR) as a nonintercepting beam-size monitor for the operational beam line. The details are provided in Ref. [18], and for completeness we show the beam line configuration in Fig. 3. An alignment laser located upstream of the station was used to adjust the mirror angles for best transmission. The first 50-mm diameter lens was at $140 \mathrm{~cm}$ from the source point giving an acceptance half angle of $18 \mathrm{mrad}$. The electron-beam energy was $4.5 \mathrm{GeV}$ so the $1 / \gamma$ OTR lobe was at $\pm 110 \mu \mathrm{rad}$, and we obtained beam image sizes of about 125 to $300 \mu \mathrm{m}$ depending on the upstream focusing quadrupoles' settings. An Al-coated optically flat Si crystal of $300-\mu \mathrm{m}$ thickness was used as the converter screen for this series of OTR data taken as reference for the ODR data. A wire harp was located in close $z$ proximity to the OTR/ODR station and served as an 


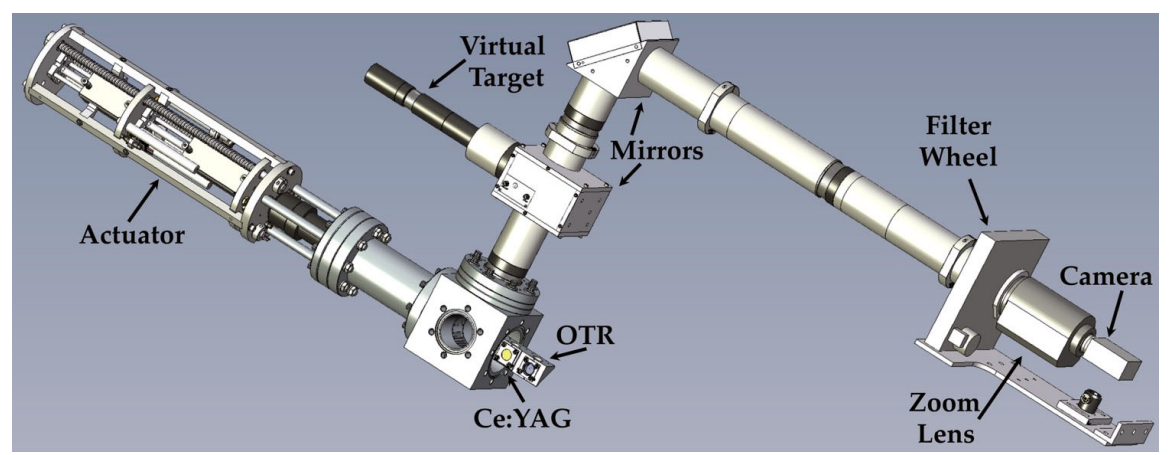

FIG. 2. The Fermilab prototype station with cube and actuator, screens, virtual target, optics, filter wheels, zoom lens, and camera.

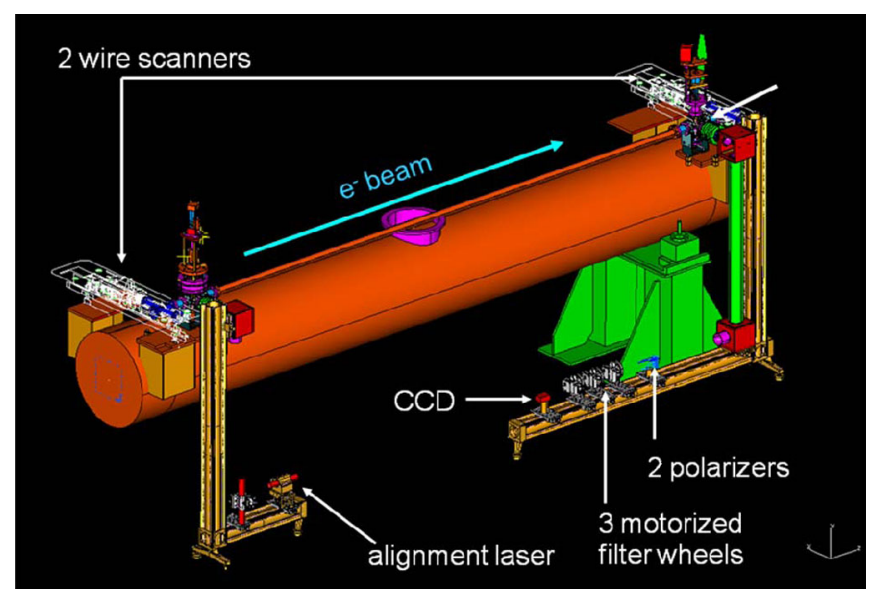

FIG. 3. A schematic of the OTR/ODR station on the beam line at JLAB showing the optical transport, the CCD camera, the alignment laser, and the wire scanner locations [18].

independent beam-size monitor for low-power beam. The camera was a JAI-A60 CCD with a National Instruments 10-bit frame grabber/digitizer. We note the linear polarizers were on a holder on a flipper actuator so they provided a $25-\mathrm{mm}$ diameter aperture when inserted and no aperture when withdrawn. The calibration factors were about $10.9 \mu \mathrm{m} /$ pixel in the $x$ and $y$ directions.
The tests at the Advanced Photon Source (APS) at Argonne National Laboratory (ANL) were performed in the Booster ring extraction (BTX) line which provided 7-GeV single micropulses containing up to $3.2 \mathrm{nC}$ each at $2 \mathrm{~Hz}$. A schematic is shown in Fig. 4 that indicates the OTR/ODR Al metal screen was rotated around the vertical axis azimuthally at 45 degrees to the beam direction on a vertical stepper-motor-driven actuator. The details of the OTR/ODR station are provided elsewhere [17] and predated the tests at JLAB and Fermilab. The optics included a first lens of $50-\mathrm{mm}$ diameter with $1-\mathrm{m}$ focal length at a distance of $1 \mathrm{~m}$ (acceptance of $25 \mathrm{mrad}$ half angle) and relay optics with filter wheels providing the options for the linear polarizers or neutral density filters. The lobe opening angle for the OTR is $\sim 70 \mu \mathrm{rad}$. These were the first reported near-field focusing tests with ODR, and we referenced our images to the observed OTR beam image sizes at the same screen location when the screen was inserted fully and intercepted the beam. Because of the larger beam sizes and field of view used at APS, calibration factors of 55 and $45 \mu \mathrm{m}$ per pixel for $x$ and $y$ axes, respectively, were employed at $7 \mathrm{GeV}$. In the course of the ODR tests, we observed significant polarization impacts on the images as predicted by the analytical model [17]. The online image processing indicated polarization effects in the OTR as well, but we have now recently also postprocessed those

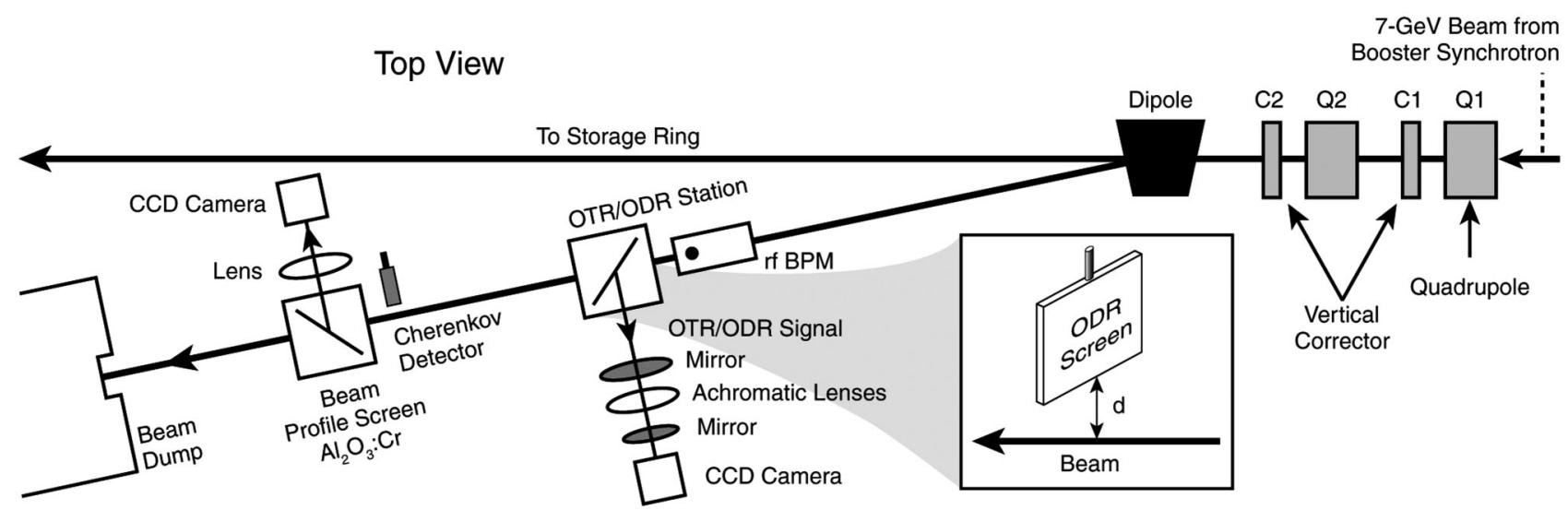

FIG. 4. A schematic of the OTR/ODR station at the 7-GeV APS BTX beam line [17]. 
same images individually using an offline Gaussian fitting routine to evaluate statistical errors more carefully on the sets of ten images in each configuration.

\section{OTR POINT-SPREAD-FUNCTION MODEL}

The fundamental OTR mechanism occurs when a charged particle beam transits the interface between two
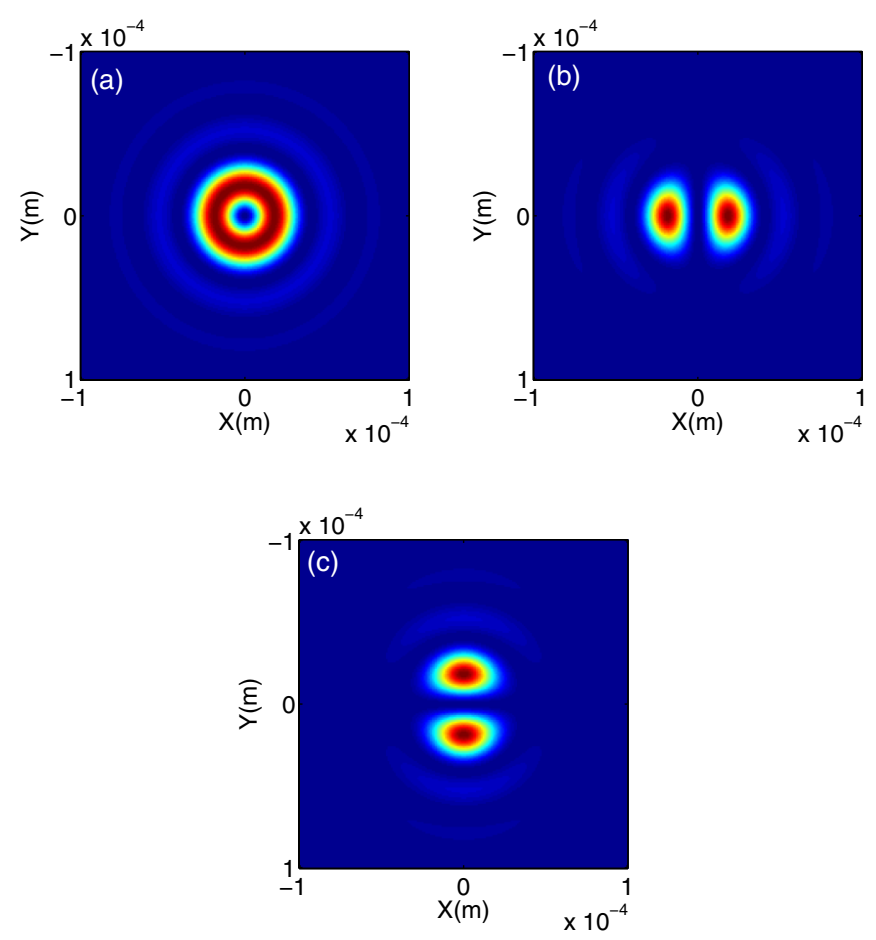

FIG. 5. Calculated OTR PSF images with (a) total, (b) horizontal polarization, and (c) the vertical polarization of the case: energy $=14.3 \mathrm{MeV}, M=1, \lambda=500 \mathrm{~nm}$, and $\theta_{\max }=0.010$.

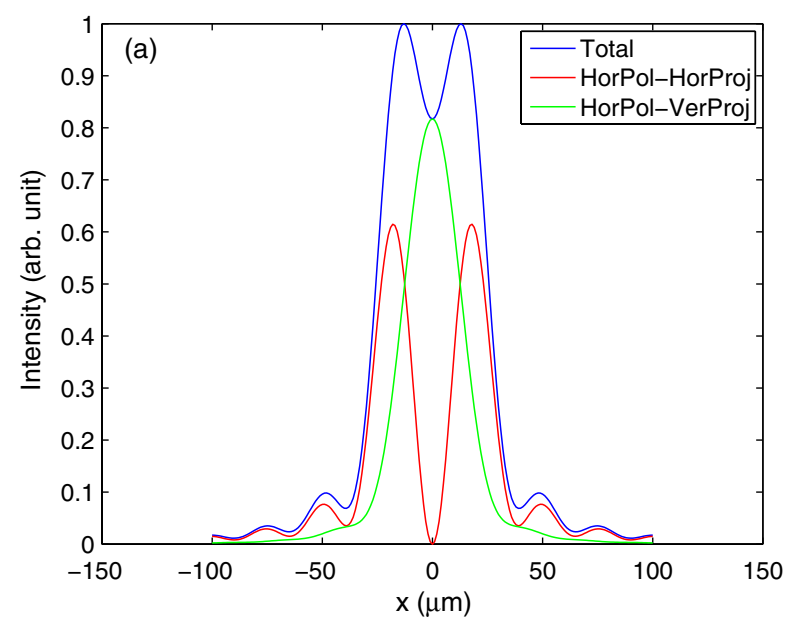

media [1,2]. The approaching charge and the induced image charge in the second medium may be treated as a collapsing dipole with the consequent emission of radiation, i.e. OTR. The yield is about one visible photon per 100 electrons incident, but they are emitted in the few-fs time scale as compared to the slower 80 -ns scintillation process. The radiation is emitted around the angle of specular reflection for backward radiation and around the angle of the beam direction in the forward direction for high gamma beams. For an oblique incidence such as 45 degrees, backward OTR is emitted at 90 degrees to the beam direction. This geometry is compatible with most accelerator beam-profiling stations.

The assessment of the actual OTR PSF has to first order been described by several authors previously [5-9]. The model invokes the convolution of the basic OTR singleparticle angular distribution function with the $J_{1}$ ordinary Bessel function to describe the intensity pattern at the detector. The concept is to calculate the electric field distribution at the image plane and then square it to get the photon intensity distribution $I(x, y)$ expected. This is described in Refs. [6-9] and shown in the expression below for a single ideal lens:

$$
I(x, y) \propto\left[\int_{0}^{\theta_{\max }} \frac{\theta^{2}}{\theta^{2}+1 / \gamma^{2}} J_{1}\left(\frac{k \theta \sqrt{x^{2}+y^{2}}}{M}\right) d \theta\right]^{2},
$$

where $k=2 \pi / \lambda$ with $\lambda$ the wavelength of radiation, $x$ and $y$ are the spatial coordinates, $M$ is the magnification, the angle of OTR emission is $\theta$, and the Lorentz factor is $\gamma$. The angle of integration is limited by the aperture of the lens $\left(\theta_{\max }\right)$, and this can have a strong effect on the PSF in the model.

Because at the simplest level OTR is inherently radially polarized, the calculated point spread function does reflect

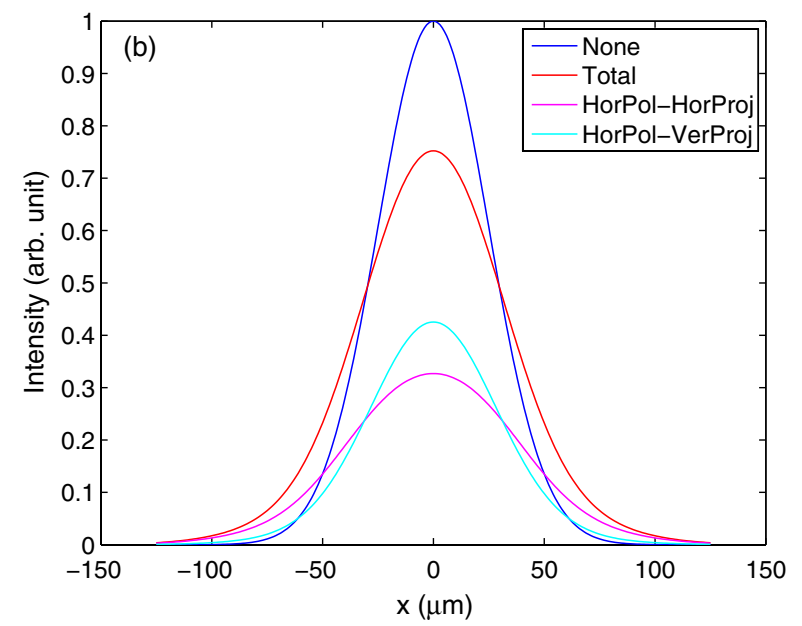

FIG. 6. (a) OTR PSF projections of the images in Fig. 5 with total, horizontal polarization with horizontal projection, and the vertical projection of the same case. (b) Comparison plots of the original $x$ size $(25 \mu \mathrm{m})$ with no convolution and convolutions with the OTR PSF projections of the total $(33.1 \mu \mathrm{m})$, horizontal polarization-horizontal projection $(37.7 \mu \mathrm{m})$, and the vertical projection $(29.3 \mu \mathrm{m})$ of the same latter case. 

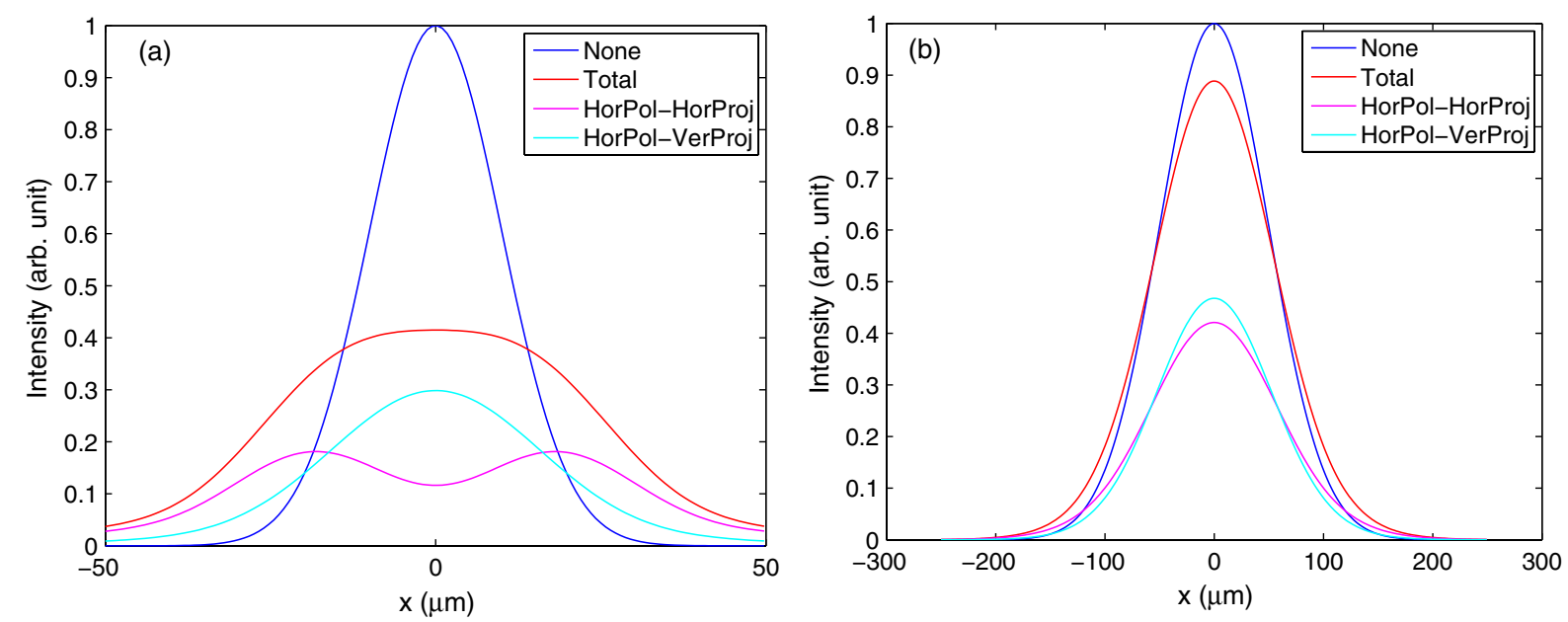

FIG. 7. Comparison plots of the original $x$ size $10 \mu \mathrm{m}$ (a) and $50 \mu \mathrm{m}$ (b) with no convolution and with convolutions. The 50- $\mu \mathrm{m}$ case was convolved with the OTR PSF projections of the total $(56.1 \mu \mathrm{m})$, horizontal polarization-horizontal projection $(59.2 \mu \mathrm{m})$, and the vertical projection $(53.4 \mu \mathrm{m})$ of the same latter case.

this aspect in the upper left image of Fig. 5. Note the axes scales are $\pm 100 \mu \mathrm{m}$. The horizontal polarization component appears as a double lobe as seen in Fig. 5(b), while the vertical polarization is seen also as a double lobe in Fig. 5(c). These are not far-field angular distribution patterns, but the PSF in the image plane. The projections of these are shown overlaid in Fig. 6(a) where the total (blue curve), horizontal polarization with horizontal projection (red curve), and horizontal polarization with vertical projection (green curve) exhibit different features.

We next show in Fig. 6(b) the results of convolving the initial beam size of $\sigma=25 \mu \mathrm{m}$ with the OTR PSF projections of Fig. 6(a). We have compared test convolutions of the PSF and the initial beam size in 2D space with convolutions of the respective projections and, as expected, the results are identical.

The results of the Gaussian fits to the convolved profiles are: total $=33.1 \mu \mathrm{m}$, horizontal polarization $=$ $38.0 \mu \mathrm{m}$, and the vertical projection of the horizontal polarization $=29.3 \mu \mathrm{m}$. Since our experimental data fit well to Gaussian profiles (as expected for the high gamma beams extracted from a recirculating accelerator configuration), for consistency we also used Gaussians in our model. The model does support the concept that use of the perpendicularly polarized component is closer to the original $25 \mu \mathrm{m}$ size than using the total or parallel components. Even in this vertical projection case one would still need to deconvolve the PSF to get the actual beam size. Note this several-micron effect was generated for illustrative purposes only by using the 10-mrad acceptance angle, which is 2 times smaller than for the high-gamma experiment optics and a 2 times smaller beam size. The results in Fig. 7 for convolving an initial $50-\mu \mathrm{m}$ beam size and the OTR PSF using the same optical parameters as in Fig. 5 are: total $=56.1 \mu \mathrm{m}$, horizontal polarization $=59.2 \mu \mathrm{m}$, and vertical projection $=53.4 \mu \mathrm{m}$. The effects are relatively smaller and symmetrically change around the total value. In this case the $2.7-\mu \mathrm{m}$ reduction in the convolved total OTR profile sigma versus the perpendicular one is indicated, and both are larger than the initial beam size. For a $100-\mu \mathrm{m}$ initial beam size for the same parameters, the effect would be even smaller and even more difficult to detect in practice.

A more extreme case is described in Ref. [21], where the beam size in the vertical plane is only $2.2 \mu \mathrm{m}$, and the polarized PSF's double lobe for their optical system with magnification of 18 is actually visualized in the image such as indicated in the 10- $\mu \mathrm{m}$ case shown in Fig. 7(a). Those authors used the visibility of the valley between the lobes to deduce the small beam sizes.

\section{EXPERIMENTAL RESULTS}

\section{A. 14.3-MeV case (Fermilab)}

The fundamental issue is whether one can detect a measurable difference in beam-profile sizes if one uses the perpendicular component of OTR, and if so, what is the magnitude? We used focusing by upstream quadrupoles to generate narrow vertical and horizontal stripes at the prototype station. Examples of the images are shown in Fig. 8. The total OTR is at the left and the vertically polarized at the right. The Gaussian fits are used on the projected profiles from the region of interest and are shown below each image. In this case they are $12.6 \pm$ 0.06 and $10.4 \pm 0.20$ pixels, respectively. The results are tabulated in Table II. The digital camera gain was adjusted to balance the signal levels being processed. Ten-image individual averages were done with a MATLAB-based image processing program, and the average variance was divided by $10^{1 / 2}$. We see an $\sim 12-\mu \mathrm{m}$ reduction (18\%) in the initial $67-\mu \mathrm{m} x$ size when using 

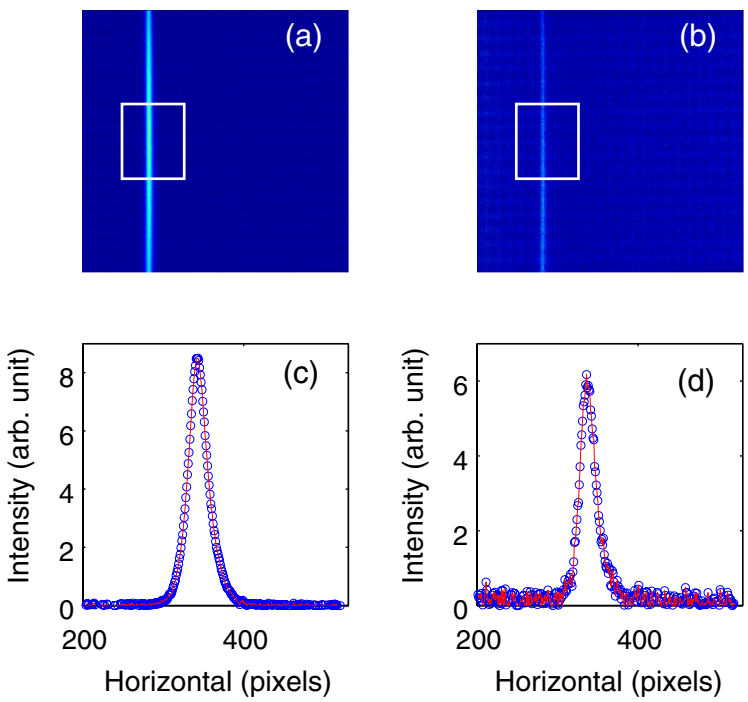

FIG. 8. OTR images of vertical stripes (a) total OTR and (b) vertically polarized OTR. The projected $x$ profiles from the region of interest are shown below each image.

the perpendicular (vertical polarization) OTR component as compared to the two independent data sets taken with no polarizer.

Examples of the horizontal images are shown in Fig. 9. The total OTR is at the left and the horizontally polarized OTR image at the right. The Gaussian fits are used on the projected $y$ profiles from the region of interest and are shown below each image. In this case they are $11.8 \pm$ 0.11 and $10.5 \pm 0.12$ pixels, for $y$ sizes of total and horizontally polarized, respectively.

The vertical size results are tabulated in Table III. The digital camera's gain was adjusted to balance the signal levels being processed, and the same image processing was done. We see an $\sim 7-\mu \mathrm{m}$ reduction $(11 \%)$ in the initial $62-\mu \mathrm{m} y$ size with the horizontally polarized OTR component. This is based on averaging the three sets of data for each polarization state including a sequence change which are provided in Table III for completeness. We note that at this low gamma of $\sim 30$ with the screen rotation around a vertical axis, the horizontal polarized angular pattern is asymmetric in lobe intensity and shape while the vertical component lobes are symmetric. The broken symmetry in one plane is not in the model described in the next section, but is consistent with the smaller effect in the horizontal polarized component compared to Table II.

TABLE II. Summary of vertical-stripe data. The vertical $(V)$ polarization data are indicated in determining the $x$ sizes with the statistical uncertainty. The calibration factor was $5.3 \mu \mathrm{m}$ per pixel.

\begin{tabular}{lcc}
\hline \hline Polarization & $X$ sigma (pixel) & $X$ sigma $(\mu \mathrm{m})$ \\
\hline No & $12.6 \pm 0.06$ & $66.8 \pm 0.3$ \\
$V$ & $10.4 \pm 0.20$ & $55.1 \pm 1.1$ \\
No & $12.7 \pm 0.07$ & $67.3 \pm 0.4$ \\
\hline \hline
\end{tabular}
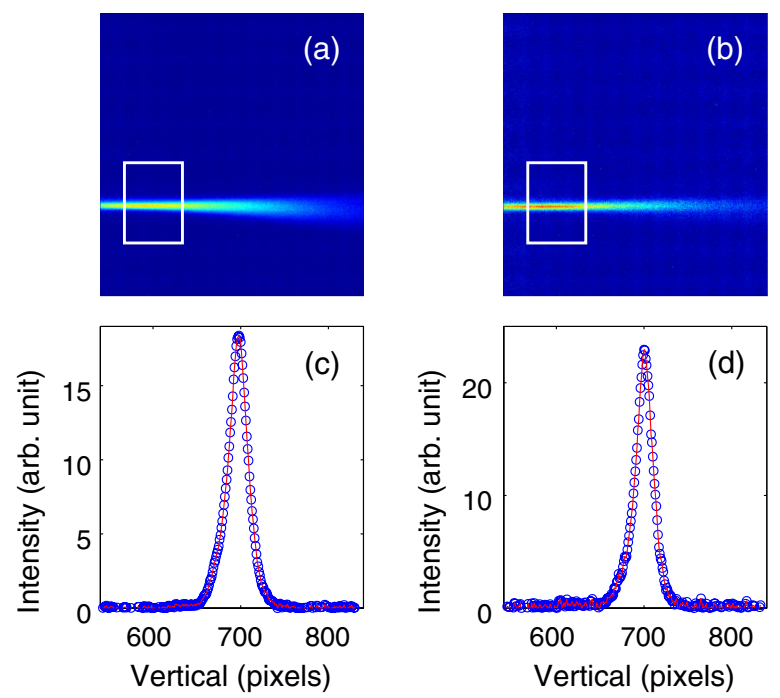

FIG. 9. OTR images of horizontal stripes (a) total OTR and (b) horizontally polarized OTR. The projected $y$ profiles from the region of interest are shown below each image, (c) and (d) respectively.

\section{B. 4.5-GeV case (CEBAF/JLAB)}

The results at CEBAF on OTR polarization effects have been reported previously within the ODR paper [18], but we include one figure for completeness here since there was a reference to the harp monitor data and have reprocessed the images for variance evaluations. These image sizes are an order of magnitude smaller than the $7-\mathrm{GeV}$ cases of the next subsection in the major axis of the elliptical beam and at a similar gamma. The actual shape of the images changed from basically round (149 $\mu \mathrm{m}$, $157 \mu \mathrm{m})$ with no linear polarizer to elliptical with the major axis parallel to the linear polarization axis selected and with the perpendicular minor axis being the dimension of interest. It is evident in Fig. 10 that the perpendicularly polarized component results are $\sim 15 \%$ smaller than the total OTR $x$ results and lie closer to the wire scan results over the range of $x$ beam sizes from 380 to $160 \mu \mathrm{m}$. The error bars indicate the standard deviation of the mean value on the ten samples which included the machine's beamsize fluctuations during data acquisition. This observed

TABLE III. Summary of horizontal-stripe data. The horizontal $(H)$ polarization data are indicated in determining the $y$ sizes. The calibration factor is $5.3 \mu \mathrm{m}$ per pixel.

\begin{tabular}{lcc}
\hline \hline Polarization & $Y$ sigma (pixel) & $Y$ sigma $(\mu \mathrm{m})$ \\
\hline No & $11.8 \pm 0.11$ & $62.5 \pm 0.6$ \\
$H$ & $10.5 \pm 0.10$ & $55.7 \pm 0.6$ \\
No & $11.5 \pm 0.10$ & $61.0 \pm 0.6$ \\
$H$ & $10.5 \pm 0.10$ & $55.7 \pm 0.6$ \\
$H$ & $10.2 \pm 0.09$ & $54.1 \pm 0.5$ \\
No & $11.6 \pm 0.12$ & $61.5 \pm 0.6$ \\
\hline \hline
\end{tabular}




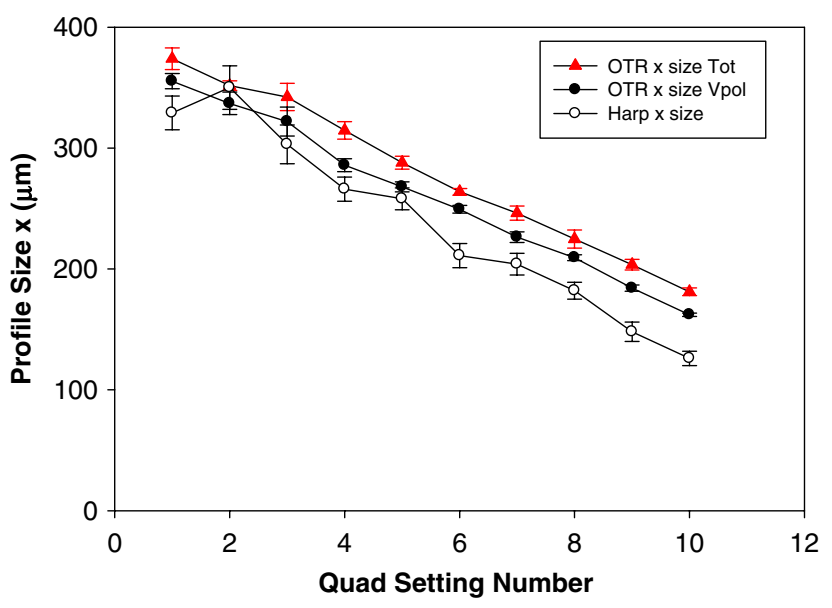

FIG. 10. Comparison of the total OTR projected profiles in $x$, vertically polarized (Vpol) OTR results, and the wire harp results as a function of upstream quadrupole (Quad) settings at $4.5 \mathrm{GeV}$ [16].

image-size reduction is consistent with the hypothesis on the inherent advantage of the perpendicular polarization component of OTR.

In addition, the low-ellipticity beam shapes in the 4.5-GeV data permitted comparison of the beam's $y$ image size for the three OTR polarization cases as shown in Fig. 11. In this case, the parallel component (Vpol) is systematically larger than the total OTR projected case values, and the perpendicular case (Hpol) has the smaller values. This is qualitatively like the PSF model in Sec. III results, except the magnitude of the differences is much larger than $2-3 \mu \mathrm{m}$, and the beam size is almost 2.5 times larger than the test case of $50 \mu \mathrm{m}$, albeit with the parameters of that section.

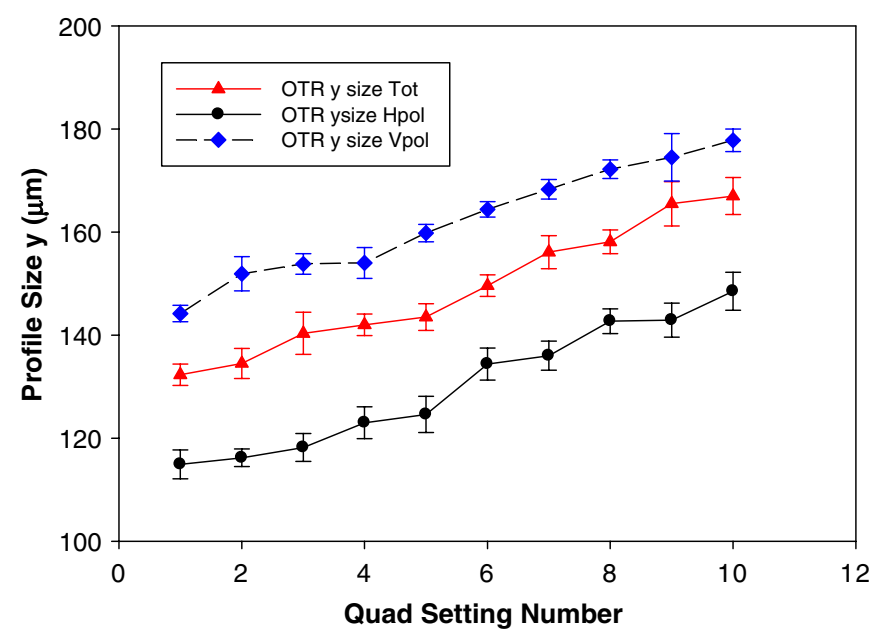

FIG. 11. Comparison of the total OTR projected profiles in $y$ (red), horizontally polarized (Hpol) results (black), and vertically polarized (Vpol) OTR results (blue) as a function of upstream quadrupole (Quad) settings at $4.5 \mathrm{GeV}$.
TABLE IV. Summary of 7-GeV APS OTR data with $3.2 \mathrm{nC}$. The horizontal $(H)$ polarization data are indicated in determining the $y$ sizes and vice versa with the vertical $(V)$ polarization data. The first three data rows are based on ten-image sums, and then the ten images were processed individually to determine the averages and the statistical errors.

\begin{tabular}{lcccc}
\hline \hline Polarization & $\begin{array}{c}X \text { sigma } \\
(\text { pixel })\end{array}$ & $\begin{array}{c}X \text { sigma } \\
(\mu \mathrm{m})\end{array}$ & $\begin{array}{c}Y \text { sigma } \\
(\text { pixel })\end{array}$ & $\begin{array}{c}Y \text { sigma } \\
(\mu \mathrm{m})\end{array}$ \\
\hline No & 19.6 & 1078 & 5.4 & 243 \\
$H$ & 18.1 & 996 & 4.5 & 203 \\
$V$ & 16.6 & 913 & 5.4 & 243 \\
Averages & & & & \\
No & $19.2 \pm 0.24$ & $1056 \pm 14$ & $5.46 \pm 0.10$ & $245 \pm 5$ \\
$H$ & $18.3 \pm 0.29$ & $1006 \pm 17$ & $4.60 \pm 0.06$ & $207 \pm 3$ \\
$V$ & $16.7 \pm 0.15$ & $918 \pm 10$ & $5.42 \pm 0.13$ & $244 \pm 5$ \\
\hline \hline
\end{tabular}

\section{7-GeV case (APS/ANL)}

Several years ago, a first series of experiments was done on the demonstration of near-field imaging of 7-GeV beams at APS with ODR-based techniques [17]. Fundamental to these studies was the imaging of linearly polarized ODR components and referencing to the OTR image sizes at the same diagnostics station. In the course of these tests, we also recorded polarized images of OTR as tabulated in Table IV. Even though the horizontal size was over $1000 \mu \mathrm{m}$, we still noticed clear image-size reductions of $15 \%$ with the perpendicularly polarized component as shown in Fig. 12 and as now reported for the first time. The projected horizontal profiles of the ten-image sums show the total OTR (black curve) result with the vertically polarized profile (red curve) width being detectably smaller! The profile intensities and baseline were scaled and shifted, respectively, to make the direct comparison.

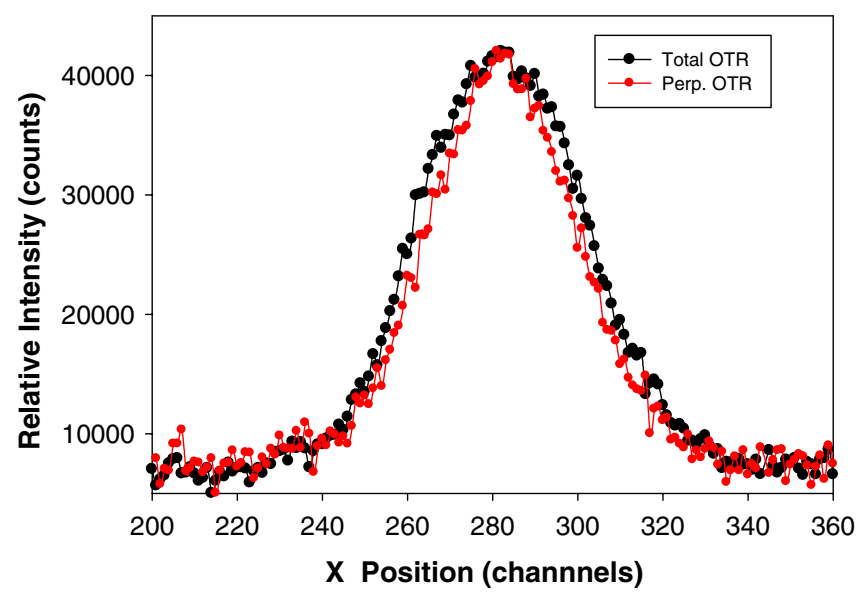

FIG. 12. Comparison of the projected OTR $x$ profiles using no polarizer (black) and the vertical polarizer (red). The perpendicular component's width is $\sim 15 \%$ smaller than the total OTR case. The beam sizes are from the ten-image sum as given in Table IV. 
The effect persisted in the smaller vertical dimension at $245 \mu \mathrm{m}$ as well with a $\sim 16 \%$ reduction to $207 \mu \mathrm{m}$. We have revisited the ten-image sets to establish the statistical uncertainty in the measurements which are included in Table IV.

\section{DISCUSSION}

If one then collects examples from the three experiments at FNAL, JLAB, and ANL, the empirical evidence indicates a systematic reduction of image sizes with the perpendicular component and basically a gamma-independent effect as seen in Table $\mathrm{V}$ where similar fractional effects were observed at $14.3 \mathrm{MeV}$ and $4.5 \mathrm{GeV}$ in the $125-\mu \mathrm{m}$ size regime $[15,18]$. In these PSF case calculations we used $\theta_{\max }=50 \mathrm{mrad}$ for the $14.3 \mathrm{MeV}$ case, and 18 and $25 \mathrm{mrad}$, respectively, for the high-gamma cases at JLAB and APS. A 700-nm wavelength was used to provide an upper limit on the bandwidth effect of broadband OTR since most of the photons sensed by the CCD are below this value and seen with more efficiency. Experimentally, we can only obtain directly the total OTR image and the linearly polarized components of it so we reference the fractional size reduction to the total OTR image size in column 2. Moreover, the profile differences scale with the beam image size so the fractional reduction is very similar at $\sim 15 \%-19 \%$ in the experimental results of columns $2-4$. Only when the PSF width is closer to the actual beam size from the polarized PSF or single-particle function of Refs. [6,7], respectively, do we calculate larger effects. We see the growth of the PSF effects at 25 and $10 \mu \mathrm{m}$ with $\theta_{\max }=10 \mathrm{mrad}$ as in Sec. III. The magnitude of the difference empirically is $4-5$ times the modeled result in general. It is consistent with the induced-current distributions being larger than the actual charge distribution. Also, the perpendicular PSF has not been deconvolved yet, so the actual beam size is even smaller presumably as indicated by the results shown in columns 4-8. In principle, the PSF could be determined quantitatively by using a delta-function-like beam size in the few- $\mu \mathrm{m}$ regime to visualize it as done by Aryshev et al. [21]. In practice, we generally cannot generate such small beams in many of our accelerators, or if we do, the charge is then too low to image readily.

As an aid to display the systematics of the convolution of the OTR PSF and Gaussian beam sizes, we show the convolved output graphically in Fig. 13 as a function of actual/initial beam size. In this case we used a 2D convolution of the PSF and beam sizes and then did the projections. In Fig. 13(a) for the $14.3 \mathrm{MeV}$ case, we used the $\theta_{\max }=50 \mathrm{mrad}, \lambda=700 \mathrm{~nm}$, and $M=1$ as described earlier for actual beam sizes from 5 to $100 \mu \mathrm{m}$. The expected decrease of the differences between the ratios at larger sizes is seen, and the perpendicular component is also always smaller than the total OTR result. However, the experimental points (diamonds) are seen to be outside of the corresponding values on the curves, i.e., data differences are anomalously larger. In Fig. 13(b) we show a similar graph of calculated values for the 4.5$\mathrm{GeV}$ case with $\theta_{\max }=18 \mathrm{mrad}$ and $M=0.8$ and for a range of actual beam sizes of 100 to $450 \mu \mathrm{m}$. The experimental data points for linear polarized perpendicular versus total OTR differences are clearly larger than the calculated values (blue curve) by more than 3 standard deviations.

We also present an assessment of possible factors to explain these differences between the model and experiment. We considered (1) actual acceptance angle, (2) actual bandwidth of OTR, (3) sensitivity of CCD sensor, (4) linearity of digital system when polarizer is installed, (5) lens imperfections, (6) OTR screen imperfections, (7) optical focusing of linearly polarized light, (8) multiple lens system vs a single lens, (9) a change of focus or aperture with a polarizer inserted, and (10) 2D convolution vs 1D in the models. Our assessments follow for each area.

(1) We have measured the prototype optics acceptance angle for the lower gamma case and reconfirmed the first

TABLE V. Summary of results from the three series of experiments ordered by increasing total OTR image sigma size. The fractional reductions obtained with the perpendicular component compared to the total OTR are 3-10 times larger in these experimental cases in column 4 than in the calculated OTR PSF convolution cases in column 8.

\begin{tabular}{|c|c|c|c|c|c|c|c|}
\hline \multicolumn{4}{|c|}{ Experimental results } & \multicolumn{4}{|c|}{ OTR PSF model convolution results } \\
\hline $\begin{array}{l}\text { Beam } \\
\text { energy } \\
(\mathrm{GeV})\end{array}$ & $\begin{array}{l}\text { Total OTR } \\
\text { sigma } \\
(\mu \mathrm{m})\end{array}$ & $\begin{array}{l}\text { Perpendicular } \\
\text { OTR sigma: } \\
x, y(\mu \mathrm{m})\end{array}$ & $\begin{array}{l}\text { Fractional } \\
\text { size } \\
\text { reduction }(\%)\end{array}$ & $\begin{array}{l}\text { Initial } \\
\text { sigma } \\
(\mu \mathrm{m})\end{array}$ & $\begin{array}{c}\text { Total OTR } \\
\text { sigma } \\
\text { calculation }(\mu \mathrm{m})\end{array}$ & $\begin{array}{c}\text { Perpendicular } \\
\text { OTR sigma } \\
\text { calculation }(\mu \mathrm{m})\end{array}$ & $\begin{array}{l}\text { Calculated } \\
\text { size } \\
\text { reduction }(\%)\end{array}$ \\
\hline 0.014 & 67 & 55 & 18 & 50 & 50.9 & 50.5 & 1 \\
\hline 0.014 & $125^{\mathrm{a}}$ & $101^{\mathrm{a}}$ & 19 & 100 & 100.8 & 100.4 & 0.4 \\
\hline 4.5 & 149 & 124 & 17 & 125 & 141 & 134 & 5 \\
\hline 4.5 & 157 & 130 & 17 & 125 & 141 & 134 & 5 \\
\hline 7.0 & 245 & 207 & 16 & 200 & 211 & 206 & 3 \\
\hline 7.0 & 1078 & 913 & 15 & 1000 & 1020 & 1011 & 1 \\
\hline
\end{tabular}

${ }^{\mathrm{a}}$ Ref. [19]. 

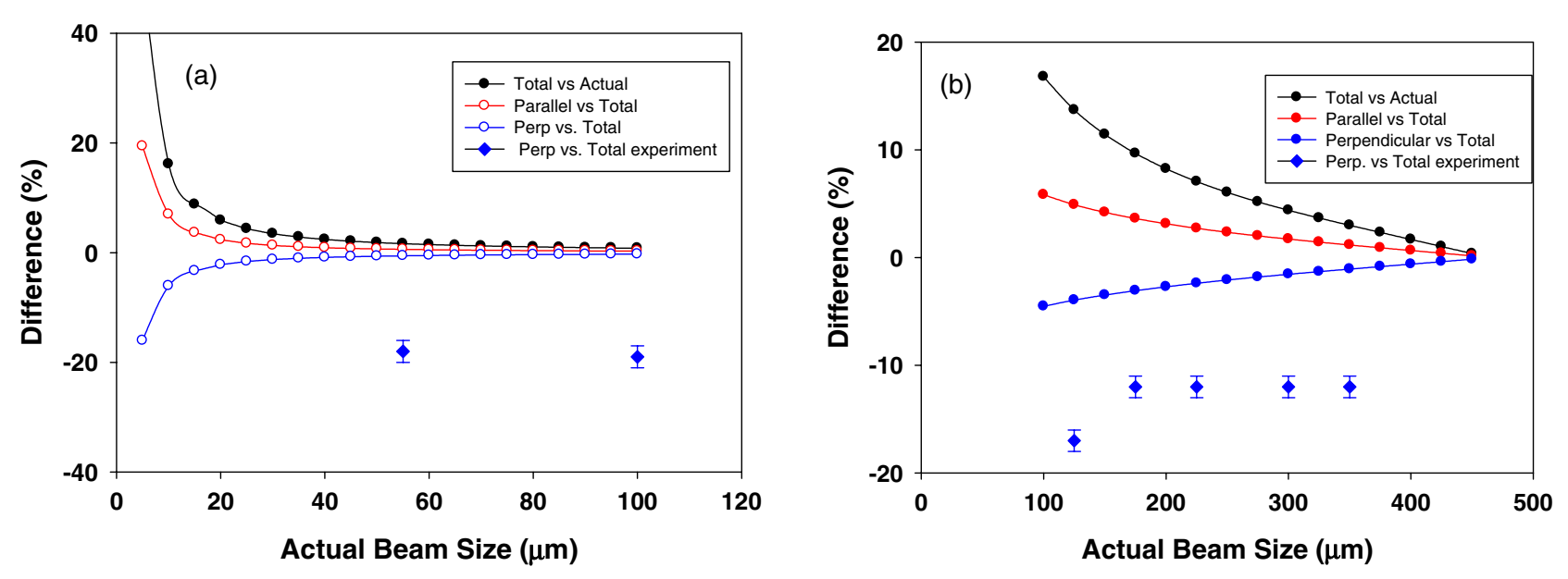

FIG. 13. Results of comparing the OTR PSF model component ratio results with different actual initial beam sizes for (a) 14.3-MeV case and (b) 4.5-GeV case. The experimental results for linear polarized orthogonal or perpendicular OTR vs the total OTR (diamonds) lie outside the model curves.

lens position of the two high-gamma tests. These are 50, 18 , and $25 \mathrm{mrad}$ for $14.3 \mathrm{MeV}, 4.5 \mathrm{GeV}$, and $7 \mathrm{GeV}$ cases, respectively, and used for the calculated values in Table V.

(2) There is a calculable wavelength effect which we addressed by taking a longer wavelength case $(700 \mathrm{~nm})$ in the model in Table $\mathrm{V}$ where there is reduced sensitivity in the CCD sensor compared to the effective average wavelength in the experiment.

(3) The CCD sensor has the standard Si response which peaks in the $550-\mathrm{nm}$ regime, is $50 \%$ less at $700 \mathrm{~nm}$, and is falling in value rapidly as one goes to longer wavelengths. The glass faceplate of the CCD generally limits signal to wavelengths longer than $\sim 380 \mathrm{~nm}$. No filters were used in the experiment so we integrated over the visible light regime. In the model we took a long wavelength $(700 \mathrm{~nm})$ to illustrate a worse case.

(4) The polarizer reduces light transmitted, and the digital processing may underestimate the size. We evaluate this as a small effect and note the lack of any detectable polarization effect with YAG : Ce scintillator light taken with the same system as OTR [19], and the clear increase of the beam size with the parallel component in the JLAB data with the initially roundish beam spot. Three different digitizers were used at the three setups.

(5) Although we do not have ideal lenses and mirrors, all OTR components went through the same set of lenses, and in two cases a clear glass was used to compensate the optical path for the inserted polarizer material.

(6) OTR screens were optically flat Si substrates with Al coating at Fermilab and JLAB, and a polished Al mirror at APS. All supported a laser alignment procedure where an upstream laser beam was transported through the optical system. Only minor effects were observed.

(7) There is a minor advantage in optical focus for a specific transverse dimension for any linearly (and perpendicular) polarized light transiting a lens compared to unpolarized light. Although qualitatively in the correct sense, the slight reduction in image size in an optical system by only a few wavelengths in magnitude with use of the linearly polarized light perpendicular to a dimension of interest as described in Mansuripur's book [22] is discounted for explaining the much larger experimental effects observed in this study.

(8) In practice, we used multiple lens transport while the model considers a single lens case. There were achromat lenses in some positions. In the experiments, all polarization components went through the same optics, but the effect persisted.

(9) Actually, with a polarizer on the flipper actuator, the aperture is the same or smaller when inserted so the PSF could only be larger and the observed image larger. This is opposite to the effect observed for the perpendicular component.

(10) We have compared test convolutions of the PSF and the initial beam size in 2D space with convolutions of the respective projections and, as expected, the results are identical. In addition the Table V entries are 2D PSF projections before convolution with the beam size and the plots in Fig. 13 are the full 2D convolution of the PSF and the axisymmetric beam Gaussian. The conclusions are the same.

Based on these assessments the data exhibit larger than expected (anomalous) differences in the OTR polarized image sizes, and they are consistent with a predominant linear polarization effect, probably in the time-averaged induced-current distribution.

Additionally, in near-field optical diffraction radiation (ODR) experiments at high gamma at APS and JLAB, one had observed a similar pattern where the perpendicular component always resulted in a narrower ODR image in the $x$ plane. The linear polarizers were installed for the ODR tests with OTR images to be used as a reference. One existing model for ODR is reported in [17], and it clearly shows significant differences between the parallel and 


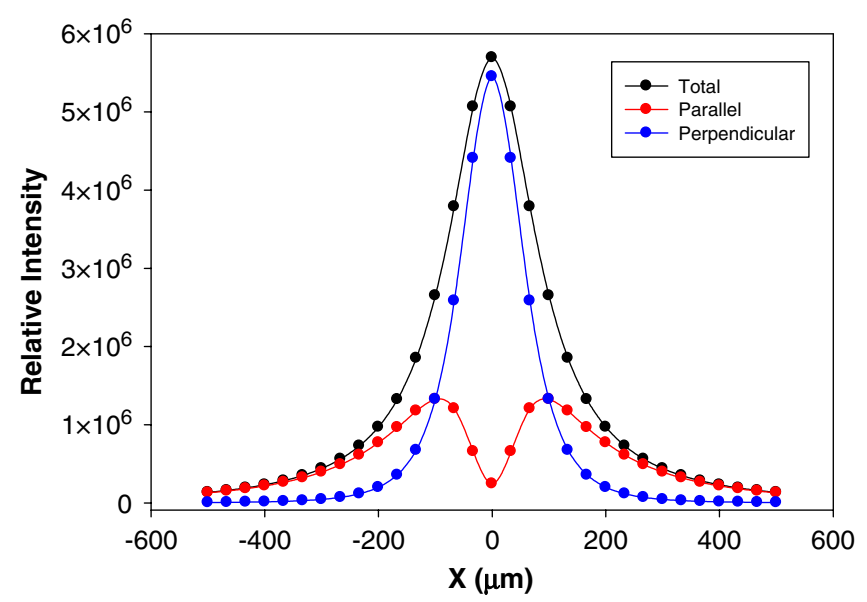

FIG. 14. Comparison of different ODR polarization components with relative intensities: total (black), perpendicular (blue), and parallel (red). The actual Gaussian beam size is $20 \mu \mathrm{m}$, the height is $20 \mu \mathrm{m}$, and the impact parameter is $100 \mu \mathrm{m}$ in the simulation [23].

perpendicular polarization components computationally for the transverse profiles and as observed experimentally $[17,18]$. Since a single plane screen whose edge was positioned above the beam by $\sim 1 \mathrm{~mm}$ was used, and thus broke vertical symmetry, the vertically polarized component was a single lobe and the parallel polarization was a weaker-in-intensity double lobe as shown in Fig. 14. This simulation is at $7 \mathrm{GeV}$ for a $20-\mu \mathrm{m}$ sigma initial beam size in $x$ and $y$ and the 100- $\mu \mathrm{m}$ impact parameter [23]. We routinely used the vertically polarized ODR images that were smaller in the $x$ dimension than the total ODR image for monitoring $x$-beam size. We also acquired the reference OTR images without and with polarizers inserted.

\section{SUMMARY}

In summary, we have detected linear-polarizationdependent profile sizes for OTR images of relativistic electron beams. Generally, the magnitude of the imagesize reduction with the perpendicular polarization component compared to the total OTR is several times larger than predicted by the standard OTR PSF models [7,9] (and thus termed anomalous). These polarization effects were observed over a wide range of gamma from $\sim 30-14000$ and beam sizes from $\sim 50$ to $1000 \mu \mathrm{m}$. The magnitude of the experimental effect is only approached in the PSF model by reducing the solid angle subtended by the optics and reducing the beam size. However, the simple convolution of the PSF and beam size does not explain the systematic differences at beam sizes $>100 \mu \mathrm{m}$, much less at $1000 \mu \mathrm{m}$. We find that the OTR polarization effects are qualitatively analogous to what were observed in ODR near-field experiments in the past [17] which may also be attributed to the timedependent induced-current distribution in the metal surface which we time average via the emitted OTR or ODR in the CCD camera. We recommend the practical use of the OTR perpendicular (orthogonal) component relative to the beam dimension at a minimum and the subsequent deconvolution of that component's contribution when known. We will continue investigations with the imaging stations on the Advanced Superconducting Test Accelerator now under construction with expected beam energies of 50 to $800 \mathrm{MeV}$. We encourage other investigators to assess such effects in their experiments including that of high dynamic range beam-halo imaging with OTR and to consider additional modeling of the basic mechanism of OTR production.

\section{ACKNOWLEDGMENTS}

The authors acknowledge support from N. Eddy and M. Church and the technical assistance of J. Santucci of Fermilab. The first author also acknowledges various discussions on OTR with H. Loos and J. Frisch of SLAC, with R. Ischebeck of PSI, with R. Fiorito of the University of Maryland, and with D. Rule of NSWC. FNAL is operated by Fermi Research Alliance, LLC under Contract No. DEAC02-07CH11359 with the United States Department of Energy; ANL work was supported by the U.S. Department of Energy, Office of Science, under Contract No. DEACO2-O6CH11357; and JLAB contribution was authored by Jefferson Science Associates, LLC under U.S. DOE Contract No. DE-AC05-06OR23177 with modeling and analysis work supported by U.S. DOE office of Basic Energy Sciences under the early career program; DOE Award No. FWP\#JLAB-BES11-05.

[1] V.L. Ginzburg and I. M. Frank, Sov. Phys. JETP 16, 15 (1946).

[2] L. Wartski, S. Roland, J. Lasalle, M. Bolore, and G. Filippi, J. Appl. Phys. 46, 3644 (1975).

[3] R. B. Fiorito and D. W. Rule, in Proceedings of the 1994 Beam Instrumentation Workshop, Los Alamos NM, AIP Conf. Proc. No. 319, edited by Robert E. Shafer (AIP, New York, 1994), p. 21.

[4] A.H. Lumpkin et al., in Proceedings of the 21st Particle Accelerator Conference, Knoxville, 2005 (IEEE, Piscataway, NJ, 2005), p. 4162 [http://www.JACoW.org].

[5] V. A. Lebedev, Nucl. Instrum. Methods Phys. Res., Sect. A 372, 344(1996).

[6] X. Artru, R. Chehab, K. Honkavarra, and A. Variola, Nucl. Instrum. Methods Phys. Res., Sect. B 145, 160 (1998).

[7] M. Castellano and V. A. Verzilov, Phys. Rev. ST Accel. Beams 1, 062801 (1998).

[8] A. P. Potylitsyn, in Proceedings of the Advanced Radiation Sources and Applications Workshop, edited by H. Wiedemann (Springer, Dordrecht, Netherlands, 2006), p. 149.

[9] Dao Xiang, Wen-Hui Huang, and Yu-Zheng Lin, Phys. Rev. ST Accel. Beams 10, 062801 (2007). 
[10] M. Borland et al., in Proceedings of the Particle Accelerator Conference, Chicago, IL, 2001 (IEEE, New York, 2001), pp. 2707-2709; Nucl. Instrum. Methods Phys. Res., Sect. A 483, 268 (2002).

[11] E. L. Saldin, E. A. Schneidmiller, and M. V. Yurkov, Nucl. Instrum. Methods Phys. Res., Sect. A 490, 1 (2002).

[12] Z. Huang, M. Borland, P. Emma, J. Wu, C. Limborg, G. Stupakov, and J. Welch, Phys. Rev. ST Accel. Beams 7, 074401 (2004).

[13] R. Akre et al., Phys. Rev. ST Accel. Beams 11, 030703 (2008).

[14] A. H. Lumpkin, R. J. Dejus, and N. S. Sereno, in Proceedings of the 30th International FEL Conference (Pohang Accelerator Laboratory, Kyeongju, Korea, 2008) [http://www.JACoW.org]; Phys. Rev. ST Accel. Beams 12, 040704 (2009).

[15] A. H. Lumpkin et al., in Proceedings of the 14th Beam Instrumentation Workshop, Santa Fe, New Mexico (LANL, Santa Fe, 2010), TUPSM001 [http://www.JACoW.org].

[16] A. H. Lumpkin et al., in Proceedings of the 2011 Particle Accelerator Conference, NY, USA (IEEE, New York, 2011), MOP219 [http://www.JACoW.org].
[17] A. H. Lumpkin, W. J. Berg, N. S. Sereno, D. W. Rule, and C.-Y. Yao, Phys. Rev. ST Accel. Beams 10, 022802 (2007).

[18] P. Evtushenko, A. H. Lumpkin, A. P. Freyberger, and C. Y. Liu, in Proceedings of the Beam Instrumentation Workshop (LBNL, Lake Tahoe, CA, 2008), WECOTC01, p. 332 [http://www.JACoW.org].

[19] A. H. Lumpkin, A. S. Johnson, J. Ruan, J. Santucci, Y.-E Sun, R. Thurman-Keup, and H. Edwards, Phys. Rev. ST Accel. Beams 14, 060704 (2011).

[20] Alex H. Lumpkin, in Proceedings of the Beam Instrumentation Workshop (JLAB, Newport News, VA, 2012), TUBP02 [http://www.JACoW.org].

[21] A. Aryshev et al., in Proceedings of IPAC10, Kyoto, Japan (KEK, Tsukuba, Japan, 2010), MOPEA052, p. 195 [http:// www.JACoW.org].

[22] Masud Mansuripur, Classical Optics and its Applications (Cambridge Press, Cambridge, England, 2009), Chap. 3.

[23] C.-Y. Yao, A. H. Lumpkin, and D. W. Rule, in Proceedings of the 2007 Particle Accelerator Conference, Albuquerque, New Mexico (IEEE, New York, 2007), FRPMS001 [http:// www.JACoW.org]. 